\title{
Effect of Micronutrient Fortification on Nutritional and Other Properties of Nixtamal Tortillas
}

\author{
K. E. Burton \\ keburton5@yahoo.com \\ Frost M. Steele \\ frost_steele@byu.edu \\ Laura K. Jefferies \\ laura_jefferies@byu.edu \\ Oscar A. Pike \\ oscar_pike@byu.edu \\ Michael L. Dunn \\ Follow this and additional works at: https://scholarsarchive.byu.edu/facpub \\ Part of the Food Science Commons, and the Nutrition Commons
}

\section{Original Publication Citation}

K.E. Burton, F.M. Steele, L. Jefferies, O.A. Pike, and M.L. Dunn. "Effect of Micronutrient Fortification on Nutritional and Other Properties of Nixtamal Tortillas." Cereal Chemistry. 85.1

(28): 7-75.

\section{BYU ScholarsArchive Citation}

Burton, K. E.; Steele, Frost M.; Jefferies, Laura K.; Pike, Oscar A.; and Dunn, Michael L., "Effect of Micronutrient Fortification on Nutritional and Other Properties of Nixtamal Tortillas" (2008). Faculty Publications. 212.

https://scholarsarchive.byu.edu/facpub/212

This Peer-Reviewed Article is brought to you for free and open access by BYU ScholarsArchive. It has been accepted for inclusion in Faculty Publications by an authorized administrator of BYU ScholarsArchive. For more information, please contact ellen_amatangelo@byu.edu. 


\title{
Effect of Micronutrient Fortification on Nutritional and Other Properties of Nixtamal Tortillas
}

\author{
K. E. Burton, ${ }^{1}$ F. M. Steele,${ }^{1}$ L. Jefferies,${ }^{1}$ O. A. Pike,${ }^{1}$ and M. L. Dunn ${ }^{1,2}$
}

Nixtamalization is the process of steeping dried corn in hot water with calcium hydroxide (lime) with subsequent removal of all or most of the pericarp through washing. The resulting product is called nixtamal. Approximately $60 \%$ of corn tortillas in Mexico are produced from nixtamal, with the remainder prepared from nixtamalized corn flour. Nixtamal was fortified with micronutrient premix containing iron, zinc, folic acid, niacin, riboflavin, and thiamin. Premix composition followed a proposed Mexican regulation for corn flour fortification, adjusted for moisture. Effects of premix on masa adhesiveness, hardness, and $\mathrm{pH}$, as well as tortilla sensory properties, stretchability, rollability, and color were measured. Micronutrient levels were tested in the dry corn, nixtamal, masa, and tortillas. There were no significant differences in masa texture or $\mathrm{pH}$, tortilla rollability, or consumer acceptance of tortillas when comparing unfortified control and fortified treatments. Added thiamin was almost entirely degraded during processing. Folic acid and riboflavin decreased 26 and $45 \%$, respectively, through the masa-tortilla manufacturing process. Niacin showed no significant loss. Despite processing losses, fortification resulted in significant nutrient increases compared with control tortillas. Folic acid increased $974 \%$, riboflavin increased 300\%, niacin increased $141 \%$, iron increased $156 \%$, and zinc increased $153 \%$ in fortified tortillas.
The traditional diet among a large segment of the Mexican population is lacking in a number of key micronutrients. The National Nutrition Survey, conducted by the National Institute of Public Health of Mexico, estimated that iron and zinc deficiencies were $\approx 52$ and $33 \%$, respectively, for children under five years of age (Rivera and Amor 2003). Iron, zinc, and folic acid deficiencies were estimated to be 36,20 , and $10 \%$, respectively, for children between 5 and 11 years of age. Additionally, 41, 39, and 5\% of women ages 12-49 had iron, zinc, and folic acid deficiencies, respectively. Riboflavin deficiency is not common but has been seen in populations consuming a diet based almost exclusively on corn products (Faber et al 2001).

Corn tortillas are a staple in the Mexican diet, constituting 60$90 \%$ of cereal product intake among many parts of the population (Villalpando 2004). On average, corn tortillas represent nearly 50\% of individual energy intake in Mexico (unpublished data). Fortification of corn tortillas would therefore be an excellent means of reducing micronutrient deficiencies in the Mexican population.

Corn tortillas can be made from nixtamal or corn flour. Nixtamal is produced by nixtamalization, which is the process of cooking and steeping dried corn in hot water with calcium hydroxide (lime). Nixtamal is wet-milled to form masa, which is baked to produce nixtamal corn tortillas. Nixtamal can also be dried and ground to make masa flour.

In an effort to reduce dietary micronutrient deficiencies, the Comisión Federal para la Protección Contra Riesgos Sanitarios (COFEPRIS) (Federal Commission for Protection Against Sanitation Risks), an agency of the Mexican government, has proposed fortification guidelines for corn masa flour (CMF) (Turner and Tirado 2003). However, CMF tortillas make up only $\approx 40 \%$ of total corn tortilla consumption in Mexico. The bulk of tortillas consumed are made from freshly ground nixtamal (unpublished data). Micronutrient fortification of traditional tortillas made from nixtamal, coupled with fortification of CMF, could improve the nutritional status of a significant percentage of the Mexican population.

\footnotetext{
${ }^{1}$ Department of Nutrition, Dietetics and Food Science, Brigham Young University, S-221 ESC, Provo, UT 84602.

${ }^{2}$ Corresponding author. Phone: 801.422.6670. Fax: 801.422.0258. E-mail address: Michael_dunn@byu.edu
}

doi:10.1094/CCHEM-85-1-0070

(c) 2008 AACC International, Inc.
The effect of micronutrient fortification on nixtamal tortillas has been evaluated in several previous studies. Figueroa et al (2003) incorporated vitamins and minerals into nixtamalized corn tortillas before the milling stage and evaluated the effect of fortification on masa and tortillas. However, nutrient increases from fortification were relatively small compared with the very high premix addition rates indicated in their study. Tovar and LariosSaldaña (2005) investigated iron and zinc addition during nixtamalization. The minerals were mixed with the lime and added during the cooking step, which is not ideal. After cooking and steeping, the aqueous phase is drained and the nixtamal is typically washed several times with fresh water before milling, which results in significant losses of added micronutrients. Actual increases in iron and zinc were 5-10 times lower than expected theoretical increases. Consumer sensory data, an important aspect of overall acceptability, was not reported in either of these studies.

Rosado et al (2005) measured vitamin and mineral losses during conversion of fortified corn masa flour into tortillas. They found that added riboflavin and thiamin incurred losses of 37 and $35 \%$, respectively. Mineral losses reported were not significant. A thorough assessment of the effects of micronutrient fortification on nutritional, sensory, and other properties of commercially produced nixtamal tortillas is needed. The objectives of this research were to evaluate these properties in commercially prepared masa and tortillas prepared from micronutrient fortified nixtamal.

\section{MATERIALS AND METHODS}

\section{Materials}

A micronutrient premix in dry powder form was provided by DSM Nutritional Products Mexico S.A. de C.V. (Mexico, DF, Mexico). Premix composition was based on vitamin and mineral levels proposed by COFEPRIS for addition to corn flour (G. Arroyo, personal communication), with adjustments made to account for the higher moisture content of nixtamal (Table I). The premix included a maltodextrin carrier that was incorporated to allow dosing of $1 \mathrm{~g}$ of premix $/ \mathrm{kg}$ of nixtamal, as well as $0.1 \%$ silicon dioxide as a flow agent. Ferrous fumarate $(7.46 \% \mathrm{w} / \mathrm{w}$ in premix), zinc oxide $(2.91 \%)$, nicotinamide $(2.02 \%)$, thiamin mononitrate $(0.36 \%)$, riboflavin $(0.17 \%)$, and folic acid $(0.13 \%)$ were used as micronutrient sources.

Corn was commercial whole-kernel, white dent corn (Honeyville Food Products, Rancho Cucamonga, CA), with a thousand kernel weight average of $319 \mathrm{~g}$. Lime was Food Chemicals Codex grade calcium hydroxide (Mississippi Lime, St. Genevieve, MO). 


\section{Commercial Nixtamalization and Sample Collection}

Nixtamal and tortillas for this study were prepared at a commercial tortilleria in Taylorsville, UT, using the tortilleria's standard manufacturing practices. Unfortified nixtamal was prepared from two different lots of dried corn on separate days. Dried corn, water, and lime in a 1:3:0.01 (w/w) ratio were combined in an open tank and heated gently for $\approx 1 \mathrm{hr}$. The mixture was manually stirred periodically during cooking. The corn was then steeped for 16-20 hr without application of additional heat. After steeping, the cooked corn (nixtamal) was transferred to a holding tank where it was washed and cooled with running tap water for $5 \mathrm{~min}$. The liquid was then drained from the nixtamal.

On each of two days, a portion of the nixtamal was split into two $32-\mathrm{kg}$ batches of unfortified nixtamal, which were used to produce two treatments: 1) control (no premix) or 2) micronutrient premix addition. Unfortified control $(\mathrm{Ct})$ nixtamal was prepared by mixing $32 \mathrm{~kg}$ of nixtamal in the tortilleria's paddle-blender for $2 \mathrm{~min}$. Micronutrient fortified (MF) nixtamal was prepared in the same way with addition of micronutrient premix (32 g) distributed evenly throughout the mixer. Mixing time was based on previous homogeneity testing using iron as a marker.

After mixing in the paddle blender, each batch of nixtamal was conveyed to the mill hopper and milled into masa dough using traditional volcanic mill stones. During milling, additional water was added by the mill operator as needed to provide an appropriate texture of masa for tortilla making and to reduce masa temperature. This is standard practice in the nixtamal tortilla industry (Rooney and Serna-Saldivar 2003). Masa was manually kneaded as it came out of the mill to form a cohesive mass and to ensure masa homogeneity. The resulting mass was transferred to a tortilla-forming machine (maquinas tortilladoras, Celorio, Naucalpan, México), which consisted of a mixer, extruder, and former in one unit. Formed tortillas were baked for 35-40 sec in a triplepass, gas-fired oven heated to $240^{\circ} \mathrm{C}$. While in the oven, tortillas were inverted twice.

Masa and tortilla samples (250 g) were collected at the beginning, middle, and end of each treatment run. Baked tortillas were stacked and allowed to sit at ambient temperature for 10-15 min before packaging in plastic sample bags and storing on ice. Tortillas were transported to the laboratory on ice and placed in a $-20.0^{\circ} \mathrm{C}$ freezer for $48 \mathrm{hr}$ before shipping frozen by overnight courier to Medallion Laboratories (Minneapolis, MN) for micronutrient analysis. Micronutrient analyses were typically completed within 12 days of sample collection. Masa samples were collected as uncooked tortilla disks immediately upon exiting the tortilla former and were treated in the same fashion as the tortillas. Masa and tortilla samples for texture and color analysis were transported back to the laboratory on ice and evaluated the same day they were produced. Tortillas were allowed to equilibrate to room temperature before reading.

\section{Masa Evaluation}

Hardness and adhesiveness. Masa hardness and adhesiveness were determined with a TA-XT2 texture analyzer (Texture Technologies, Scarsdale, NY) using methods provided by the manufacturer for tortilla doughs. Eighteen masa samples, three each from beginning, middle, and end of run on each of two days were analyzed for each treatment. To measure hardness, a $1.00 \pm 0.05 \mathrm{~g}$ masa sample was weighed and molded into a disk $1.8-1.9 \mathrm{~cm}$ in diameter and $0.3-0.4 \mathrm{~cm}$ in height. The sample of masa was placed on the texture analyzer platform. A TA 4 38.1-mm acrylic cylinder was positioned at $20.00 \mathrm{~mm}$ above the platform. The cylinder compressed the masa disk within $1.00 \mathrm{~mm}$ of the platform. The test speed was $3.0 \mathrm{~mm} / \mathrm{sec}$ and the post-test speed was 10.0 $\mathrm{mm} / \mathrm{sec}$. Hardness was defined as the maximum force required to flatten the masa sample.

For adhesiveness, a $2.00 \pm 0.05 \mathrm{~g}$ masa sample was weighed and molded into a disk $1.8-1.9 \mathrm{~cm}$ in diameter and $0.6-0.7 \mathrm{~cm}$ in height. A TA 4 38.1-mm acrylic cylinder started descent at 55.00 $\mathrm{mm}$ above the platform. The cylinder applied a constant force of $150 \mathrm{~g}$ on the masa sample for $10 \mathrm{sec}$. The test speed and the posttest speed were the same as for hardness. Adhesiveness was defined as the maximum force needed to pull the cylinder away from the masa sample.

$p H$. Masa $\mathrm{pH}$ was measured directly, without addition of water, using a $\mathrm{pH}$ meter (Orion perpHect LogR, model 320, Thermo Electron Corporation, Round Rock, TX) calibrated with $\mathrm{pH} 7$ and 10 buffer standards.

\section{Baked Tortilla Evaluation}

Texture. Rollability and stretchability tests were used to evaluate the texture of the baked tortillas. For rollability testing, a commonly used method of Arámbula et al (1999) was followed. A method of Mao et al (2002) was followed to measure tortilla stretchability. In this study, the distance the TA probe traveled was changed from 60 to $20 \mathrm{~mm}$. As with masa, 18 tortilla samples (three each from beginning, middle, and end of run on each of two days) were analyzed for each treatment.

Color. Tortilla color was determined with a spectrophotometer (Hunter Color Flex, Hunter Associates Laboratory, Reston, VA). An $18 \times 18 \mathrm{~cm}$ glass plate was placed on the colorimeter's measuring port, followed by two stacked tortillas, double-baked side down. The tortillas were held in place using another glass plate. Each set of two tortillas was read three times, moving the tortillas from left to right in a straight line (Waliszewski et al 2004). Average CIE $L^{*}$ (light-dark), $a^{*}$ (green-red), and $b^{*}$ (blue-yellow) colors were reported for each sample. Eighteen sets of tortilla samples (three each from beginning, middle, and end of run on each of two days) were analyzed for each treatment.

Sensory testing. A central-location consumer test took place at two Utah markets that cater to the local Latino community. A total of 51 expatriate Mexican $(n=47)$ and Central American $(n=4)$ consumers who regularly consume corn tortillas were recruited to evaluate sensory properties of the tortillas. Consumer panelists consisted of 25 males and 26 females, predominantly between 19 and 40 years of age, in the lower to lower-middle socio-economic class. Panels were conducted outdoors on separate days for each location between the late morning and early afternoon hours.

Overall liking, color, aroma, flavor, and texture of the tortillas were rated by panelists using a 9-point hedonic scale, with $1=$ "dislike extremely", 5 = "neither like nor dislike", and $9=$ "like extremely." Tortillas were held refrigerated $\left(4.0^{\circ} \mathrm{C}\right)$ for two to three days before the consumer test. Before serving, the tortillas were reheated on a gas-fired, cast iron griddle for $20-25 \mathrm{sec}$ on each side at $180^{\circ} \mathrm{C}$. The reheating method is a modification of a standard method from the Instituto Nacional de Nutrición Salvador Zubiran (INNSZ) (National Institute of Nutrition) in Mexico.

Tortillas from each treatment were presented using a randomized complete block design. Samples prepared on different days were represented equally. Questions were delivered and responses recorded on a paper ballot written in Spanish. The samples were

TABLE I

Micronutrient Addition Levels in Nixtamal Based on COFEPRIS Proposal for Corn Masa Flour ${ }^{\mathrm{a}}$

\begin{tabular}{lcc}
\hline Micronutrient & $\begin{array}{c}\text { Fortification of Corn } \\
\text { Masa Flour } \mathbf{( m g / k g )}\end{array}$ & $\begin{array}{c}\text { Fortification of } \\
\left.\text { Nixtamal }^{\mathbf{b}} \mathbf{( m g} / \mathbf{k g}\right)\end{array}$ \\
\hline Iron & 40.0 & 23.11 \\
Zinc & 40.0 & 23.11 \\
Folic acid & 2.0 & 1.16 \\
Niacin & 35.0 & 20.22 \\
Riboflavin & 3.0 & 1.73 \\
Thiamin & 5.0 & 2.89 \\
\hline
\end{tabular}

a Arroyo (2004).

${ }^{\mathrm{b}}$ Conversion based on estimated average of $90 \%$ solids content in flour and $52 \%$ solids content in nixtamal. 
presented in sequential monadic format on paper plates labeled with 3-digit blinding codes. Panelists were instructed to use an unsalted cracker and filtered water to cleanse the palate between samples. Data was later entered into software (Compusense five v4.6, Guelph, ON, Canada) and then exported to SAS for statistical analysis. The study was approved by the University Institutional Review Board for Human Subjects and panelists provided informed consent. Panelists received monetary compensation for their time.

\section{Nutritional Evaluation}

Dry corn, nixtamal, masa, and tortilla samples were packaged in plastic sample bags and shipped frozen to an external analytical laboratory for nutritional analysis. Iron, zinc, folic acid, niacin, riboflavin, and thiamin were analyzed in each of two composite samples from each day's production. Subsets of tortillas were reheated on a griddle at $180^{\circ} \mathrm{C}$ for $20 \mathrm{sec}$ on each side to mimic consumer reheating; these were also sent for nutritional analysis. The nutritional profile of the tortillas after reheating indicates the extent of nutritional change resulting from in-home use. It also indicates the actual nutritional benefit for the consumer. Iron and zinc were not reported in masa or reheated tortillas because it was not expected that mineral content for these samples would differ from results in tortillas as calculated on a dry basis. Moisture was also measured in each sample to calculate nutrient values on a dry basis, which allowed for direct comparison of results between samples from different stages of the process.

The external analytical laboratory used AOAC methods (AOAC 2000) for mineral and vitamin analysis. Iron and zinc were determined according to 990.08.32. Samples for folic acid analysis were prepared according to 960.46 and measured using 992.05. Samples for riboflavin analysis were prepared according to 960.46 and measured according to 940.33. Thiamin was measured with 942.23. Samples for niacin analysis were prepared according to 960.46 and analyzed using 944.13 . Moisture was tested by 925.09 and 926.08 .

\section{Statistical Analyses}

Differences between $\mathrm{Ct}$ and MF treatment means for masa properties and tortilla texture, color, and sensory attributes were assessed using the dependent $t$-test. Differences in vitamin and mineral content during the nixtamalization process and among $\mathrm{Ct}$, $\mathrm{MF}$, and MF reheated tortillas were analyzed using analysis of variance (ANOVA); comparison of means was performed accord-

TABLE II

Hardness, Adhesiveness, and pH of Control and Micronutrient-Fortified Masa ${ }^{\mathrm{a}}$

\begin{tabular}{lccc}
\hline Treatment $^{\mathbf{b}}$ & Hardness $(\mathbf{g})$ & Adhesiveness $(\mathbf{g})$ & $\mathbf{p H}$ \\
\hline $\mathrm{Ct}$ & $12,297 \mathrm{a}$ & $80.5 \mathrm{a}$ & $8.9 \mathrm{a}$ \\
$\mathrm{MF}$ & $13,878 \mathrm{a}$ & $93.1 \mathrm{a}$ & $9.0 \mathrm{a}$ \\
$\mathrm{SE}^{\mathrm{c}}$ & 1,298 & 6.53 & 0.04 \\
\hline
\end{tabular}

${ }^{a}$ Values followed by the same letter within columns are not significantly different $(P>0.05)$.

b Treatments include control (Ct) and micronutrient fortified (MF) samples.

${ }^{c} \mathrm{SE}$, standard error of the mean. ing to Tukey-Kramer using the Statistical Analysis System v.9.1 (SAS Institute, Cary, NC). A significance value of $P=0.05$ was used to distinguish significant differences among treatments.

\section{RESULTS AND DISCUSSION}

Masa and Tortilla Chemical, Physical, and Sensory Properties

There was no significant difference in masa hardness, adhesiveness, or $\mathrm{pH}$ between the $\mathrm{Ct}$ and MF treatments (Table II). Premix addition also had no significant effect on tortilla rollability (Table III). However, tortilla stretchability significantly decreased with the addition of micronutrient premix. Premix is composed of vitamins, minerals, a maltodextrin carrier, and a small amount of silicon dioxide as a flow agent. The maltodextrin makes up $\approx 80 \%$ of the premix but $<0.08 \%$ of the masa. It is unlikely that this level of carrier would significantly affect tortilla texture. Mohammad and Hallab (1973) did find adverse effects on wheat dough and bread quality upon addition of $>0.011 \%$ iron in the form of sulfate, gluconate, or trichloride. It is possible that iron, or one of the other micronutrients present in the premix, adversely affected stretchability properties of the fortified tortillas, though the exact mechanism is unclear.

Significant differences in the Hunter CIE $L^{*}, a^{*}$, and $b^{*}$ color values were found between fortified and unfortified treatments (Table III). The addition of micronutrient premix made the tortillas slightly darker and more blue-green in color. It is widely known that xanthophyll and anthoxanthin pigments in corn are affected by $\mathrm{pH}$ (Paulsen et al 2003). Millers in certain regions add additional lime to their tortillas to produce a more yellow tortilla, whereas millers in other areas wash the nixtamal more extensively to produce whiter tortillas. Tortillas produced at the mill used in this study were quite alkaline and therefore had a somewhat yellow appearance. A component elimination study with individual premix fortificants revealed that the darkening of the tortilla upon enrichment was almost entirely caused by ferrous fumarate. This compound is very dark brown in appearance and reacts with phenolic and sulfur-containing compounds in the corn to produce an even darker appearance (Hurrell 1985). The darkening effect of ferrous fumarate has also been observed in nixtamalized corn flour. Dary (2002) reported that off-colors in tortillas were observed for levels of fumarate addition $>30 \mathrm{mg}$ of $\mathrm{Fe} / \mathrm{kg}$. The significant differences in CIE values in this study are not unexpected, given the COFEPRIS recommendation of $40 \mathrm{mg}$ of $\mathrm{Fe} / \mathrm{kg}$ in the form of fumarate or sulfate. The blue-green shift could possibly result from the iron as well, though riboflavin, which is yellow-green in solution, could also have contributed. These objective differences in CIE color are important, but of greater practical concern is the effect of this change on consumer acceptance.

Despite the apparent color difference in tortillas, consumer sensory test results indicated no significant differences in hedonic scores between $\mathrm{Ct}$ and MF treatments for any of the attributes tested (Table IV). MF tortillas were found to be comparable to $\mathrm{Ct}$ tortillas in overall acceptability and other sensory attributes. Of particular interest is the fact that the instrumental color difference did not significantly affect consumer acceptance of tortillas when presented in sequential monadic fashion. It is possible that a sideby-side evaluation might produce different results. However, the

TABLE III

Texture (Stretchability and Rollability) and Color (CIE $L^{*}, a^{*}$, and $\left.b^{*}\right)^{\text {a }}$ of Control and Fortified Corn Tortillas ${ }^{b}$

\begin{tabular}{|c|c|c|c|c|c|}
\hline Treatment & Stretchability (kg) & Rollability & $\operatorname{CIE} L^{*}$ & CIE $a^{*}$ & CIE $b^{*}$ \\
\hline $\mathrm{Ct}$ & $0.34 \mathrm{a}$ & $1.0 \mathrm{a}$ & $69.5 \mathrm{a}$ & $0.36 \mathrm{a}$ & $22.6 a$ \\
\hline $\mathrm{MF}$ & $0.25 b$ & $1.1 \mathrm{a}$ & $67.5 b$ & $0.02 b$ & $20.4 b$ \\
\hline $\mathrm{SE}^{\mathrm{c}}$ & 0.03 & 0.1 & 0.42 & 0.09 & 0.22 \\
\hline
\end{tabular}

${ }^{\text {a }} L^{*}, 0=$ black, $100=$ white; $a^{*}$, negative values indicate green, positive values indicate red; $b^{*}$, negative values indicate blue, positive values indicate yellow.

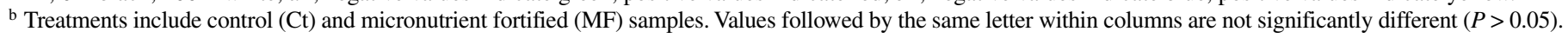

c SE, standard error of the mean. 
sequential monadic testing is more in keeping with typical consumer purchase practices.

\section{Effect of Fortification on Nutritional Properties}

Alkaline nixtamalization of corn dramatically affects the $\mathrm{pH}$ of the masa and resulting tortillas and can significantly alter the nutritional properties of these products because many vitamins are affected by changes in $\mathrm{pH}$. Ball (2006) reports that thiamin and riboflavin are stable in acidic media but become increasingly unstable at alkaline $\mathrm{pH}$, especially at elevated temperature. Folic acid, on the other hand, is quite stable in the range of $\mathrm{pH} 5-12$, but unstable below pH 5 (Ball 2006). Although minerals are elemental in nature and do not degrade, reduced bioavailability of minerals such as iron can occur at alkaline $\mathrm{pH}$ (Hurrell 1999). Due to differing consumer preferences and increasing use of acidic preservatives by certain manufacturers, commercial tortillas now cover a wide $\mathrm{pH}$ range from moderately acidic ( $\mathrm{pH} 5.5)$ to alkaline ( $\mathrm{pH}$ 8.5-9) (Ortiz and Carrillo 1997). The tortillas produced at the mill participating in this study are in the higher alkaline range and it was expected that micronutrient stability would be affected accordingly.

Vitamin levels in dry corn, as well as Ct and MF nixtamal, masa, and tortillas are reported in Table V. Process-related vitamin losses in $\mathrm{Ct}$ and $\mathrm{MF}$ products can be determined by comparing vitamin levels in intermediate and finished products to the initial levels in dried corn. Total loss of vitamins in processed fortified products can be determined by comparing MF tortilla micronutrient levels to the theoretical levels calculated based on actual premix addition to MF nixtamal.

Folic acid in native corn decreased by $24 \%$ (w/w) during the tortilla manufacturing process, with most of the degradation occurring during baking in the oven. Folic acid in MF tortillas showed a $33 \%(\mathrm{w} / \mathrm{w})$ loss during progression from the point of premix addition through baking, as compared with the theoretical value. This represented a statistically significant decline, comparable to the loss in the control product. Of particular interest is the fact that much of the degradation took place during mixing with the nixtamal, indicating that the warm, moist, alkaline environment had a destabilizing effect on this vitamin. Figueroa et al (2003) observed a somewhat larger loss of $46 \%(\mathrm{w} / \mathrm{w})$ in added folic acid through the masa/tortilla making process. The extent of the loss in the tortilla process is surprising given the fact that both Ball (2006) and Gregory (1996) have generalized that folic acid is quite resistant to $\mathrm{pH}$ and thermal degradation.

The most labile vitamin in the present study proved to be thiamin, which was almost completely degraded during nixtamalization. Total loss of native thiamin following tortilla baking was $88 \%$ (w/w). The dramatic loss of thiamin is supported by the observation that thiamin is extremely labile in alkaline conditions such as those occurring during nixtamalization (Gregory 1996). Thiamin loss in MF tortillas was $91 \%$ (w/w), as compared to theoretical. Consequently, no significant increase in thiamin resulted from fortification during this study. As indicated by samples of fortified nixtamal collected directly after mixing, most of the thiamin $(81 \% \mathrm{w} / \mathrm{w})$ was lost in the initial mixing step, as noted previously with folic acid. Bressani et al (1958) also found significant losses of thiamin when evaluating nixtamalized corn tortillas prepared in a household setting. During nixtamalization and baking, losses of native thiamin reached $62 \%(\mathrm{w} / \mathrm{w})$ in their study. Figueroa et al (2003) showed only a $36 \%$ (w/w) loss in added thiamin during processing. Rosado et al (2005) showed similar thiamin losses (35\% w/w) to Figueroa et al (2003) during conversion of fortified masa flour into tortillas.

Niacin is one of the few micronutrients that show favorable effects from nixtamalization. During the traditional corn tortilla manufacturing process, niacin is reportedly released from complex derivatives called niacytin, resulting in improved measurement and bioavailability (Heathcote et al 1952; Gregory 1996). Niacin, which is considered extremely stable in most types of cooking matrices and preparation conditions (Gregory 1996; Ball 2006), showed excellent stability in both control and fortified products in this study, with no losses being observed. Figueroa et al (2003)

TABLE IV

Mean 9-Point ${ }^{a}$ Hedonic Scores for Reheated Control and Fortified Corn Tortillas ${ }^{b}$

\begin{tabular}{lccccc}
\hline Sample & Overall Acceptability & Color & Aroma & Flavor & Texture \\
\hline $\mathrm{Ct}$ & $7.4 \mathrm{a}$ & $7.6 \mathrm{a}$ & $7.5 \mathrm{a}$ & $7.3 \mathrm{a}$ & $7.4 \mathrm{a}$ \\
$\mathrm{MF}$ & $7.3 \mathrm{a}$ & $7.3 \mathrm{a}$ & $7.5 \mathrm{a}$ & $7.2 \mathrm{a}$ & 0.26 \\
$\mathrm{SE}$ & 0.25 & 0.23 & 0.22 & 0.20 & \\
\hline
\end{tabular}

a 1 = dislike extremely, $5=$ neither like nor dislike, $9=$ like extremely.

b Treatments include control $(\mathrm{Ct})$ and micronutrient fortified (MF) samples. Values followed by the same letter within rows are not significantly different $(P>0.05)$.

${ }^{\mathrm{c}} \mathrm{SE}$, standard error of the mean.

TABLE V

Micronutrient Content of Dried Corn and Control vs. Fortified Nixtamal, Masa, and Tortillas ${ }^{\mathrm{a}}$

\begin{tabular}{|c|c|c|c|c|c|c|}
\hline Sampleb & Folic Acid $(\mu \mathrm{g} / 100 \mathrm{~g})$ & Thiamin (mg/100 g) & Niacin (mg/100g) & Riboflavin (mg/100g) & $\mathrm{Fe}(\mathrm{mg} / \mathbf{1 0 0 g})$ & Zn (mg/100g) \\
\hline Corn & $22.65 a$ & $0.45 \mathrm{c}$ & $1.31 \mathrm{a}$ & $0.16 \mathrm{a}$ & $2.34 \mathrm{a}$ & $2.30 \mathrm{a}$ \\
\hline \multicolumn{7}{|l|}{ Nixtamal } \\
\hline $\mathrm{Ct}$ & $20.84 a$ & $0.06 \mathrm{ab}$ & $1.55 \mathrm{a}$ & $0.08 \mathrm{a}$ & $2.23 \mathrm{a}$ & $2.88 \mathrm{a}$ \\
\hline MF & $201.59 d$ & $0.12 b$ & $5.37 \mathrm{~b}$ & $0.34 b$ & $7.36 \mathrm{~b}$ & $7.91 b$ \\
\hline \multicolumn{7}{|l|}{ Masa } \\
\hline $\mathrm{Ct}$ & $24.23 \mathrm{a}$ & $0.07 \mathrm{ab}$ & $2.27 \mathrm{a}$ & $0.09 \mathrm{a}$ & - & - \\
\hline MF & $189.16 \mathrm{c}$ & $0.11 \mathrm{ab}$ & $6.13 b$ & $0.38 b$ & - & - \\
\hline \multicolumn{7}{|l|}{ Tortilla } \\
\hline $\mathrm{Ct}$ & $17.28 \mathrm{a}$ & $0.06 \mathrm{a}$ & $2.46 \mathrm{a}$ & $0.08 \mathrm{a}$ & $2.68 \mathrm{a}$ & $2.50 \mathrm{a}$ \\
\hline $\mathrm{MF}$ & $185.60 \mathrm{bc}$ & $0.06 \mathrm{a}$ & $5.93 b$ & $0.32 b$ & $6.87 \mathrm{~b}$ & $6.32 b$ \\
\hline MF reheated & $179.69 b$ & $0.06 \mathrm{a}$ & $5.74 b$ & $0.35 b$ & - & - \\
\hline Theoretical $^{\mathrm{c}}$ & 276.80 & 0.64 & 5.56 & 0.42 & 6.82 & 7.65 \\
\hline $\mathrm{SE}^{\mathrm{d}}$ & 2.00 & 0.01 & 0.37 & 0.02 & 0.47 & 0.46 \\
\hline
\end{tabular}

${ }^{a}$ Results reported on a dry basis. Values followed by the same letter within columns are not significantly different $(P>0.05)$.

${ }^{\mathrm{b}} \mathrm{Ct}$, unfortified control; MF, micronutrient fortified; MF tortilla reheated on griddle.

${ }^{c}$ Theoretical value in MF tortilla based on addition of premix to Ct nixtamal with no process loss.

d SE, standard error of the mean. 
TABLE VI

Vitamin $^{\mathrm{a}}$ and Mineral Levels in 50-g Serving of Nixtamal Corn Tortillas ${ }^{\mathrm{b}}$ Compared with Daily Recommended Nutrient Intake Listed in Official Mexican Standard NOM-086-SSA1-1994c

\begin{tabular}{|c|c|c|c|c|c|c|}
\hline & Folic Acid ( $\mu \mathrm{g})$ & Niacin (mg) & Riboflavin (mg) & Thiamin (mg) & Iron (mg) & Zinc (mg) \\
\hline Ct tortilla & 8.64 & 1.10 & 0.04 & 0.03 & 1.34 & 1.25 \\
\hline MF tortilla & 89.80 & 2.87 & 0.175 & 0.03 & 6.87 & 6.32 \\
\hline Recommended & $200^{\mathrm{d}}$ & 20 & 1.7 & 1.5 & 15 & 15 \\
\hline
\end{tabular}

a Vitamin results for MF tortillas from reheated tortillas. All results reported on wet basis.

b Treatments include control $(\mathrm{Ct})$ and micronutrient premix fortified $(\mathrm{MF})$ samples.

c NOM-051-SCFI-1994 is the current standard for food labeling; $50 \mathrm{~g}$ is official Mexican serving size for tortillas.

${ }^{\mathrm{d}}$ Current FAO/WHO recommendation is $400 \mu \mathrm{g} /$ day (FAO/WHO 1998).

also reported good stability of niacin during tortilla manufacturing, with a directional $(8 \% \mathrm{w} / \mathrm{w})$ net increase in niacin through the process. Bressani et al (1958), however, showed significant losses of native niacin $(32 \% \mathrm{w} / \mathrm{w})$ in corn during preparation of nixtamal tortillas in a household setting.

Native riboflavin showed a loss of $50 \%$ (w/w) in converting dried corn to control tortilla. This loss was not surprising considering riboflavin degrades in alkaline conditions despite good retention during heat processing (Gregory 1996). Riboflavin in MF tortillas showed a loss of $24 \%$ (w/w) during processing, as compared with theoretical addition levels. In contrast, during the study by Figueroa et al (2003), riboflavin decreased $80 \%$ during processing. Rosado et al (2005) measured process losses during conversion of fortified masa flour into tortillas. They found that riboflavin decreased by $37 \%(\mathrm{w} / \mathrm{w})$, which is comparable to total losses reported in this study. Bressani et al (1958) reported $42 \%$ $(\mathrm{w} / \mathrm{w})$ loss of riboflavin during nixtamalization and baking in the home.

Despite losses in added vitamins during processing, fortification of nixtamal did result in micronutrient increases in the finished tortillas. Table $\mathrm{V}$ shows that there was a significant increase in folic acid, niacin, and riboflavin in the MF tortillas compared with the $\mathrm{Ct}$ tortillas. However, as noted previously, there was not a significant increase in thiamin. The vitamin content in the MF reheated tortillas is also shown in Table V. Only small changes in nutrient levels occurred during reheating, indicating that it is not a significant cause of nutrient loss.

Because they are elements, iron and zinc would not be expected to degrade due to thermal or chemical processing. However, losses due to leaching during steeping and washing could certainly be possible. Table $\mathrm{V}$ shows that iron and zinc levels in native corn were not affected by nixtamalization or other steps in the tortilla manufacturing process. The content of these minerals in fortified tortillas was more than double the levels in control samples. Rosado et al (2005) measured mineral levels during baking of fortified masa flour into tortillas and found no significant decreases from theoretical. Figueroa et al (2003) found $>50 \%$ loss of iron during conversion of corn to nixtamal tortillas. It is not clear if their reported loss in iron was actually due to processing, or possibly caused by a problem with the analysis.

In Table VI, the vitamin and mineral contents of Ct and MF tortillas are compared with the official recommended nutrient intakes listed in the current Mexican food labeling regulations, Norma Oficial Mexicana NOM-086-SSA1-1994 (Online at www.cofepris. gob.mx/mj/documentos/nom3.htm). The average consumers in Mexico may eat up to 10 tortillas per day (unpublished data), therefore, consumers could receive a significant percentage of the recommended intake of these nutrients from fortified tortillas.

\section{CONCLUSIONS}

Even though significant differences were found in instrumental tortilla texture and color between control and fortified treatments, these differences were not substantial enough to affect hedonic ratings during consumer testing.
Overall, tortilla fortification resulted in significant increases in all of the vitamins and minerals except thiamin, showing that added folic acid, niacin, riboflavin, iron, and zinc had acceptable stability through masa/tortilla manufacturing. Effective fortification with thiamin may require excess addition or encapsulation to withstand the moist, alkaline environment.

Because fortification of nixtamal tortillas could significantly reduce micronutrient deficiencies in target populations, and be a great benefit to those who are beyond the scope of current cereal fortification programs, additional studies in commercial mills in Mexico are being undertaken to evaluate this technology in an actual production setting.

\section{ACKNOWLEDGMENTS}

This project was conducted in collaboration with SUSTAIN through a grant from the Bill \& Melinda Gates Foundation. [Fortification of corn masa flour with iron and/or other nutrients-A literature and industry experience review. Published online at www.sustaintech.org/publications/ pubm16.pdf]. We wish to express gratitude to Adela's Tortilleria for allowing the use of their tortilleria, and DSM Nutritional Products Mexico, for their advice and provision of the micronutrient premixes. We are also grateful to Salvador Villalpando of the Instituto Nacional de Salud Publica of Mexico, as well as representatives from COFEPRIS for their continuing involvement in this project.

\section{LITERATURE CITED}

AOAC. 2000. Official Methods of Analysis of Association of Official Analytical Chemists Intl. 17th Ed. The Association: Gaithersburg MD.

Arámbula, V. G., Mauricio, S. R. A., Figueroa, C. J. D., Gonzalez-Hernandez, J., and Ondorica, F. C. A. 1999. Corn masa and tortillas from extruded instant corn flour containing hydrocolloids and lime. J Food Sci. 64:120-124.

Ball, G. F. M. 2006. Vitamins in Foods. CRC Press: Boca Raton, FL.

Bressani, R., Paz y Paz, R., and Scrimshaw, N. S. 1958. Chemical changes in corn during preparation of tortillas. J. Agric. Food Chem. 6:770-774.

Dary, O. 2002. Lessons learned with iron fortification in Central America. Nutr Rev. 60:S30-S33.

Faber, M., Jogessar, V. B., and Benadé, A. J. S. 2001. Nutritional status and dietary intakes of children aged 2-5 years and their caregivers in a rural South African community. Int. J. Food Sci. Nutr. 52:401-411.

FAO/WHO. 1998. Expert consultation on human vitamin and mineral requirements. Vitamin and mineral requirements in human nutrition: The Report of a joint FAO/WHO expert consultation. FAO/WHO: Bangkok, Thailand.

Figueroa, J. D. C., Godinez, M. G. A., Méndez, N. L. V., Guzmán, A. L., Acosta, L. M. F., and González-Hernández, J. 2001. Fortification and evaluation of nixtamalized tortillas. Arch. Latinoam. Nutr. 51:293-302.

Gregory, J. F. 1996. Vitamins. Pages 531-616 in: Food Chemistry. 3rd Ed. O. R. Fennema, ed. Food Sci. Technol: New York.

Heathcote, J. G., Hinton, J. J. C., and Shaw, B. 1952. The distribution of nicotinic acid in wheat and maize. Proc. R. Soc. London Series B. Biol. Sci. 139:276-287.

Hurrell, R. F. 1985. Nonelemental sources. Pages 39-51 in: Iron Fortification of Foods. F. M. Clydesdale and K. L. Wiemer, eds. Academic Press: Orlando, FL.

Hurrell, R. F. 1999. Iron. Pages 54-93 in: The Mineral Fortification of Foods. R. F. Hurrell, ed. Leatherhead Publishing: London.

Mao, Y. F., Flores, R. A., and Loughin, T. M. 2002. Objective texture 
measurements of commercial wheat flour tortillas. Cereal Chem. 79:648-653.

Mohammad, N., and Hallab, A. H. 1973. Effect of iron enrichment of flour on the dough characteristics and organoleptic qualities of Arabic bread. Pak. J. Sci. Indus. Res. 16:115-119.

Ortiz, J. J. O., and Carrillo, M. G. V. 1997. Shelf-life and sensory evaluation of corn tortillas, made with preservatives and texture conditioners. Arch. Latinoam. Nutri. 47:372-376.

Paulsen, M. R., Watson, S. A., and Singh, M. 2003. Measurement and maintenance of corn quality. Pages 159-211 in: Corn: Chemistry and Technology. P. J. White and L. A. Johnson, eds. AACC International: St. Paul, MN.

Rivera, J. A., and Amor, J. S. 2003. Conclusions from the Mexican national nutrition survey 1999: Translating results into nutrition policy. Salud Publica Mex. 45:S656-S675.

Rooney, L. W., and Serna-Saldivar, S. O. 2003. Food use of whole corn and dry-milled fractions. Pages 495-535 in: Corn: Chemistry and
Technology. P. J. White and L. A. Johnson, eds. AACC International: St. Paul, MN

Rosado, J. L., Cassís, L., Solano, L., and Duarte-Vázquez, M. A. 2005. Nutrient addition to corn masa flour: Effect on corn flour stability, nutrient loss, and acceptability of fortified corn tortillas. Food Nutr. Bull. 26:266-272.

Tovar, L. R., and Larios-Saldaña, A. 2005. Iron and zinc fortification of corn tortilla made either at the household or at industrial scale. Int. J. Vitamin Nutr. Res. 75:142-148.

Turner, E., and Tirado, A. 2003. The promise and challenge of corn masa flour fortification. World Grain 21:54-63.

Villalpando, S. 2004 Tortilla Fortification Working Group Meeting. El problema de la biodisponibildad de hierro en harina de maiz nixtamalizada. National Institute of Public Health: Mexico City.

Waliszewski, K., Estrada, Y., and Pardio, V. 2004. Sensory properties changes of fortified nixtamalized corn flour with lysine and tryptophan during storage. Plant Foods Human Nutr. 59:51-54.

[Received August 16, 2007. Accepted September 21, 2007.] 